\title{
El dilema bioético ante la iatrogenia del otro - mis colegas iserán como hermanos?
}

\author{
The bioethical dilemma facing the iatrogenesis \\ of others - will my colleagues be as equal to my brothers?
}

Alberto Campos*

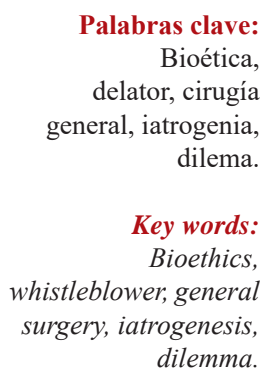

* Programa de Maestría y Doctorado en Ciencias Médicas, Odontológicas y de la Salud, Facultad de Medicina, UNAM.

Recibido: 10/10/2016 Aceptado: 14/10/2016

\section{RESUMEN}

Quien denuncia representa una amenaza para los denunciados y para él mismo, y ambas partes lo saben. El dilema consiste en saber si, por la iatrogenia misma que origina la denuncia, denunciar tiene un interés público en tanto produzca un beneficio en el sistema. Aun contemplando todas las condiciones en las que se da el hecho, y los escenarios plausibles de las consecuencias, no es posible dilucidar el efecto que la denuncia tendrá. No hay una relación entre denunciar y el resultado que se busca con esa intervención sobre una iatrogenia multifactorial. No es posible saber si una agregación de causas incidentales puede anular, mediante fenómenos emergentes, el efecto deseado. Al contrario, la denuncia, puede causar procesos autoorganizativos a partir del desorden provocado inicialmente, que produzcan efectos indeseados tanto en el denunciante como en el sistema, que aumenten la fragilidad de ambos. Es más plausible que, a la luz de consideraciones sobre las consecuencias, el agente moral, buscando anular los efectos de rebote sobre él, en lugar de resolver, disuelva el dilema y siga adelante.

\section{ABSTRACT}

The physician who blows the whistle poses a threat to the accused and to herself, and both parts know it. The dilemma consists in knowing whether, by the same iatrogenesis which originates the complaint, a report is of public interest, inasmuch as it can produce a benefice to the system. Even considering all conditions around the fact and all plausible scenarios of the consequences, it is not possible to elucidate the effect that a complaint will have. There is no relationship between reporting and the result sought with this intervention on a multifactorial iatrogenesis. It is not possible to know if an aggregation of incidental causes can override, by emerging factors, the desired effect. On the contrary, whistleblowing can cause self-organizing processes out of the disorder initially triggered which themselves can produce undesirable effects on the complainant and the system, with a final result that increases the fragility of both. It is more plausible that, in the light of considerations on the consequences, the moral agent seeking to nullify the effects on her, rather than resolve, dissolves the dilemma.

\section{INTRODUCCIÓN}

- A quién corresponde hacer un juicio sobre otro cirujano, sus técnicas y habilidades? Es una pregunta dilemática porque, de plantearse públicamente ambas partes, el crítico y el criticado, pierden; porque podemos ser, alternativamente, tanto uno como el otro; porque de no plantearse ni en lo público ni en lo privado, también ambas partes, y además los pacientes, pierden.

Pero el que sea un dilema, con todas las dificultades inherentes, no es pretexto para no abordar el problema. Echar las compresas purulentas bajo la alfombra no lo resuelve; no lo resuelve porque las más de las veces sabe- mos lo que hacemos y lo que escondemos; no lo resuelve porque la corrección política no desaparece, ni previene con sus eufemismos la iatrogenia; no lo resuelve porque al ocultar una realidad desagradable mediante muletillas fomenta la falta de análisis y el autoengaño; no lo resuelve, en fin, porque la corrección política no es correcta.

\section{PLANTEAMIENTO DEL PROBLEMA}

El dilema de denunciar a un colega iatrógeno no es del todo racional; lleva una carga emocional fuerte. El planteamiento incluye frecuentemente premisas inapropiadas y racionalizaciones que van de lo explicativo a lo 
justificatorio. Veamos algunos de los condicionales específicos de estos razonamientos y si las respuestas nos sacan del problema.

Si el cirujano no se ve objetivamente, si no tiene autoconciencia de su torpeza o si ésta se ablanda en el hacerse cirujano viejo, que tiende a ser autoindulgente y a justificar sus errores, si por tanto no sabe cuándo dejar su práctica profesional por el bien del paciente, si el cirujano es heroico porque "así es el carácter del cirujano" que muere en la raya, operando, (de hecho una falacia argumental llamada petición de principio, que pide partir de una afirmación que se da por verdadera), si el cirujano no ha contemplado, ni sabe ni puede hacer ninguna otra cosa, si nadie supera la usura del tiempo; entonces denunciarlo y exponerlo ies un acto moral o inmoral? Si pueden ser ambas cosas, entonces ¿dónde está el equilibrio del juicio moral de un cirujano? Entonces la iatrogenia del cirujano viejo, ¿es meramente un problema utilitario, un problema entre el costo y el beneficio, parecido a la curva de aprendizaje de un residente? ¿Es un mal esperado, ineludible e irremediable? Entonces, ¿dónde quedan los pacientes? Seguimos en el brete.

\section{EL TIPO DE DILEMA}

Este es un dilema de coerción, por una parte, entre códigos deontológicos explícitos o tácitos, de la sociedad y las acciones que se esperan de nosotros como miembros, y por la otra, nuestras percepciones morales, las obligaciones que nos imponemos de acuerdo con ellas, y las decisiones que habremos de tomar. Entre asentir calladamente y sin crítica con lo que los demás esperan y actuar conforme nuestra conciencia moral requiere. Entre nuestra responsabilidad profesional en el interés del paciente y la lealtad hacia nuestros colegas.

\section{DILEMAS CONTRA CÓDIGOS EXTERNOS}

Veamos en primer lugar las denuncias por malas prácticas. Éstas pueden incluir errores de aptitud, por impericia técnica o enseñanza defectuosa, en los que el cirujano lidia con su torpeza, solitario. Porque, abramos el hilo del discurso, no adquirió los conocimientos nece- sarios, porque el adiestramiento es frecuente y lamentablemente mediocre, por un provincialismo intelectual, el orgullo rural de pensar que la montaña más alta del mundo es el cerro más alto que haya visto en su pueblo, y la mejor técnica es la única técnica defectuosa, de guerra, que le enseñó algún maestro también provincial y mediocre, porque se conforma con ser submonolingüe. El provincialismo existe en los hospitales de enseñanza, ni todos tienen los recursos, ni en todos hay una perspectiva de excelencia quirúrgica; la excelencia tiene un costo. Por el contrario, el pragmatismo rural que reduce el intelecto a lo útil no mira hacia arriba, fija el rasero a la baja y paga el precio en iatrogenia. Vuelvo del excurso, delatar cirujanos ineptos tiene la consecuencia de atacar el sistema y congregarlo en contra, fenómeno que más abajo llamaré Fuenteovejuna. Otro tipo de ineptitud y torpeza es la que aumenta sin que el cirujano lo advierta claramente, mientras la decrepitud hace lo suyo; más abajo citaré a Plinio, el Viejo.

Existen también las denuncias por errores de actitud, como el fraude, cuando hay cómplices, cuando el cirujano estrella es cirujano fantasma, que no opera ni vigila a sus pacientes pero tiene operadores negros, o cuando el académico estrella tiene escritores negros, en la sombra. Por cierto, el término 'negrero' se acuñó en el s. XIX, y deriva del francés négrier, negrego, el escritor que firmaba cuando los folletines semanales requirieron producción en masa; el nègre, peyorativo de negro (y no noir), era el esclavo, quien escribía. El eufemismo 'fantasma' denota la condición de aparecido, de ilusión óptica. Uno surge como de la nada, la pieza quirúrgica en bandeja, la piyama ensangrentada, a explicar la operación que no hizo; el otro lee con impostación la conferencia que no escribió, cuya bibliografía desconoce. Ambos sostienen su prestigio en gente apta pero menor por desconocida, un negro quirúrgico o literario. Ambas estrellas hacen carrera a fuerza de retórica, de algún tipo de coerción y de la ingenuidad del otro que, por lo mismo, no denuncia; de suerte que esas situaciones, por ser circunstancias aceptadas, no constituyen un dilema. El cirujano negro o el escritor negro aceptan la coerción como ficha de cambio, mientras cultivan el propio prestigio y acceden 
a una oportunidad de reconocimiento. Otros, sin embargo, perduran a la sombra del tronco vacío y raigambre profunda y, como la hierba, perpetúan la práctica.

Ahora bien, si esa conducta es aceptada por ambas partes ¿es inmoral? Podría argumentarse que no, porque hay acuerdo, porque alguno, por razones que no son tema de esta reflexión, se conforme con menos; pero es un acuerdo bajo coerción, por lo que la aceptación de la proposición no es autónoma, no le beneficia más que parcialmente, al no reconocer su trabajo le causa daño y por tanto no es justa. Ahí está, un mínimo análisis desde el Principialismo. Alguna razón social habrá para que sólo se hable de esto como cotilleo, sotto voce; algún tipo de entendimiento anómalo. Aun otros tipos de faltas de actitud son las cirugías ficticias, procedimientos innecesarios adyacentes al acto quirúrgico para aumentar el costo o hacer manitas.

\section{DILEMAS CONTRA CÓDIGOS INTERNOS}

Éstos son más difíciles de lidiar porque son una confrontación puramente personal. La coerción es diferente, entre los valores morales adquiridos y los problemas a los que estos valores hacen frente. El agente moral puede resolver el dilema o disolverlo y abstenerse. En el primer caso, delate o no delate, el resultado es todos pierden, y será discutido más delante. El segundo caso, cuando el dilema se disuelve y no se actúa, es interesante porque entran en juego los eufemismos morales, motivos auxiliares que se usan para elegir la opción fácil. El fenómeno consiste en echar la moral bajo la alfombra mediante solidaridades gremiales. Esas solidaridades pueden darse en ambos bandos, delatores y delatados, autoridad y subordinados; para esconderse en la multitud todo vale.

Un ejemplo clásico, del Siglo de Oro, es Fuente Ovejuna, de Lope de Vega. ${ }^{1}$ Veamos. Tras la muerte del comendador Don Fernán Gómez de Guzmán a manos de la turba, los Reyes Católicos ordenan se nombre un juez "pesquisidor", pero, ante la muchedumbre, toda prueba de participación individual es imposible. Inmersos en la masa, cada componente pierde su individualidad. ${ }^{2}$ Este ejemplo de los llamados dramas municipales bien puede ser extrapolado a las morales municipales contemporáneas, un fenómeno llamado parroquialismo moral.

En efecto, quien sabe alguna iatrogenia del otro, prefiere callarla sin romper el statu quo, y con ella se hace cómplice, no sea que una pesquisa le atribuya responsabilidad individual en una iatrogenia futura. Hay diversos tipos de amenaza velada mediante falacias argumentales que se aducen para proteger al implicado; tienen entonces un doble fin. Veamos algunas; argumentum ad populum (apelación a la opinión popular) "la iatrogenia es algo muy común"; argumentum ad misericordiam (apelación a la misericordia) "no hagamos leña del árbol caído"; argumentum ad baculum, ad consequentiam (apelación a un posible castigo, a las consecuencias), "en casa del jabonero..."; argumentum secundum quid (argumento según el cual) "si al Dr. Fulano y al Dr. Zutano les pasó, a todos nos puede pasar"; "nadie es inmune a la iatrogenia" (tú tampoco). Dicho culteranamente, "errare humanum est" (errar es humano) aunque convenientemente se le ampute la segunda parte al hemistiquio "sed diabolicum est in malo perseverare" (pero es diabólico perseverar en el mal). ${ }^{3}$

\section{SOLUCIONES POSIBLES}

Hay varias, intuitivas todas. Es muy difícil hacer un cálculo puramente racional de los daños que una denuncia causaría. Veamos primero la posibilidad utópica; ¿en verdad podemos hablar con ese cirujano? ¿Será receptivo, acaso si es un joven aprendiz pero, sobre todo, si es viejo? ¿Tendrá capacidad cognitiva? ¿Se dará cuenta, cambiará, replanteará su vida, tiene alternativas?

Hay soluciones que parecerían más realistas. Ante todo, hablar con él conlleva un riesgo de enemistad. Pero quizá una segunda conversación, en el evento de una segunda iatrogenia. Quizá escalar el problema a la autoridad competente, de la manera adecuada, paso a paso. Pero habría, al mismo tiempo, que proteger el propio empleo, asegurarse de tener consenso, pues, al salir del municipio moral, los demás, en un mecanismo de autodefensa, pueden contradecir nuestra opinión, y la escalada resultar 
contraproducente. Dicho de otro modo, este es un asunto de dinámicas complejas; tener razón no garantiza la colaboración de los demás, y sí puede garantizar perder un trabajo.

Veamos entonces la relación causa-efecto; si delatar es malo para el sistema y malo para el soplón, sobre todo cuando el cirujano iatrógeno tiene una posición de prestigio, entonces ¿qué gano o qué arriesgo con mi tranquilidad moral? La solución pragmática es la disolución del dilema, la abstención. El dilema no existe. El dilema es del otro.

\section{DISCUSIÓN}

Pontificar sobre el deber moral tiene dos menoscabos. El primero es que la voluntad de imponer el punto de vista propio es inmoral. El segundo es que señalar defectos ajenos desata el encono y la réplica emocional, impugnatoria, de querer enseñar al otro lo que no se aprende, el estereotipo es la paja en ojo ajeno, bajo la forma de otra falacia argumental, el tu quoque (tú también), el rechazo al razonamiento pretendiendo la inconsistencia de quien lo hace, porque la crítica se le aplica igualmente; una variedad de ataque ad hominem (a la persona) como mecanismo de defensa, para distraer la atención y minimizar el problema. Aunque del tu quoque no se sigue que las consideraciones sobre el dilema no sean pertinentes, ni el que la iatrogenia por ser ubicua justifique el rechazo de su análisis. Con todo, estas falacias frecuentemente conducen a discusiones circulares. Entonces será más productivo plantear las reflexiones en forma de preguntas, desde ambas perspectivas, del delator y del delatado, de modo que su análisis nos sitúe críticamente frente al problema. Antes de plantearlas, y so pena de ser obvio preciso mi posición, desde dónde abordo el problema; ciertamente, he cometido iatrogenia. La afirmación no excusa las mías ni me impide hacer un análisis.

Bien. La delación requiere dos condiciones, no sólo la inmediatez de las consecuencias sino también la especificidad de la conducta para que la señal de alarma sea capaz de determinar una responsabilidad con precisión. El peligro objeto de la delación es desconocido porque es mantenido en secreto, ya por la institución ya por los miembros que están en falta; entonces, para que la denuncia sea significativa debe haber un riesgo concreto y no un presentimiento vago. En este sentido, el acto de delatar difiere de la lamentación y del augurio terrible. A pesar de todo, dadas las presiones externas sobre el delator, la mayoría de las delaciones, cuando no provocan suficientemente al auditorio, están destinadas al fracaso y el mensaje se disipa. Es por eso que la delación requiere de un contexto amplio en el que secreto, corrupción y coerción no estén atrincherados, donde el mensaje no rebote y lesione al delator. Delatar en la anarquía es asunto quijotesco. ${ }^{4}$

Así las cosas, veamos ahora si el dilema puede resolverse mediante la acción. ¿Qué tan racional es delatar la iatrogenia? ¿Qué tan racional callarla? A decir verdad, ese parroquialismo no es cuestión de moral ni de lealtad sino de miedo, miedo de ser estigmatizado por los colegas, de cometer una iatrogenia futura bajo el microscopio del otro, de las consecuencias de un error propio frente a la exacerbada atención de quien fue delatado, quien desde entonces nos llevará un registro personal. Ese parroquialismo es una versión sin garantía del dilema del prisionero, no delatar para no ser delatado. El miedo, por otra parte, es explicable; pero ¿hasta qué punto? No podría contestar eso con la precisión de una encuesta, aunque parece entendible que nadie quiera ser víctima de un ostracismo gremial implícito, que ciertamente puede afectar la vida académica. Pero, la iatrogenia del otro ien verdad afectará mi dinámica laboral, el día a día con mis pacientes? Y la pregunta anti utópica, ¿delatar la iatrogenia del otro no será agua dulce en mar salada?

Planteemos ahora las preguntas a manera de escrutinio, haciendo un movimiento pendular de los argumentos y contraargumentos hacia la inacción. Si veo el problema de soslayo y no entro al juego, disuelvo el dilema. Entonces la iatrogenia del otro ies su problema? Pues, si por omisión, quien no denuncia es cómplice, ¿dónde queda mi postura moral? ¿O 'postura moral' sólo son grandes palabras? De nuevo, ¿̇y mi empleo?, ¿̇y mi prestigio? Se hace patente entonces una tensión entre la utopía frágil por el riesgo que conlleva y el pragmatismo estable por la seguridad del silencio. Pero, ila seguridad de quién es importante, del médico? ¿O es que el pragmatismo estable no tiene consecuen- 
cias? Por una parte, la razón de la cirugía es el paciente, no el médico, no la enfermedad per se como objeto de estudio abstracto. Para eso fuimos educados io no? Para intervenir por el paciente. Pero queda el problema de las consecuencias sobre el cirujano. Veamos entonces al cirujano iatrógeno y su entorno, el medio ambiente.

No hay edad obligatoria para el retiro de un cirujano. Se habla de entre 65 y 75 años, pero ¿con qué base? A los 65 un cirujano puede ser muy apto, aunque su resistencia física a operaciones maratónicas no se compare con la de un cirujano de cuarenta. Por otra parte, el deterioro cognitivo es muy variable. ${ }^{5,6}$ Claramente, pueden contrarrestarse hasta cierto punto los temblores, la pérdida de las habilidades manuales y de la habilidad visual y espacial. ${ }^{7}$ Pero no todo es físico cognitivo, la edad sola no es la única causa de iatrogenia, también lo son la complejidad de las intervenciones y la poca frecuencia con que se realicen; entonces sí entra en juego la edad, como factor determinante. ${ }^{8} \mathrm{Y}$ algo más, el cirujano viejo suele tener una mala combinación de la falsa seguridad con base en su experiencia y de la dificultad cognitiva para adquirir conocimientos nuevos; el cirujano viejo no se actualiza. ${ }^{9}$

Veamos de nuevo la solución utópica. Tengamos en cuenta que no tenemos que ser jueces, sino ir paso a paso, comentar primero con el colega iatrógeno. Dependiendo del resultado y de casos futuros de iatrogenia de ese mismo cirujano, discutir con quienes están en posición de tomar medidas para resolver o reparar, amigablemente, el problema. Ahora bien, ¿es viable la utopía? Aceptemos que (casi) siempre habrá resistencia, que nuestra acción desatará la furia. Es entendible, sí, pero ¿justificada? ¿Qué esconde la furia? ¿No es el autoanálisis parte del profesionalismo? ¿Qué impide el autoanálisis?

Planteo ahora la refutación de la solución utópica. Nada hay peor que la utopía en tanto ideal inalcanzable, que su polo opuesto, la distopía, cuando además la realidad no se ve muy claramente, cuando la realidad es indeseada. Si el médico viejo no es feliz, ¿por qué no deja de ejercer? ¿De qué vive el médico que ya no ejerce? ¿Del mito del anciano venerable? La línea entre la imagen idealizada del anciano venerable y la imagen distópica del viejo vulgar es muy delgada. Cito ahora otro clásico, la Historia natural de Plinio el Viejo. "Natura vero nihil hominibus brevitate vitae praestitit melius." "En verdad la naturaleza no ha concedido al hombre nada mejor que la brevedad de la vida." Y sigue; "se embotan los sentidos, los miembros entorpecen, la vista, la marcha, aún los dientes y los órganos alimenticios mueren antes que nosotros." ${ }^{\prime 10} \mathrm{El}$ cirujano que envejece no es excepción de la descripción de Plinio; su percepción disminuye, es torpe de vista y de manos, resiste menos un transoperatorio largo, intenta atajos. El cirujano viejo que no se retira termina por ser distópico.

Delatar a un colega, por los efectos colaterales destructivos que supone, ha de ser siempre el último recurso, después de haber considerado otras alternativas que hayan sido rechazadas o inútiles. Antes de delatar es preciso preguntar qué peso tiene un juramento en un conflicto de lealtades. Cierto, un juramento es una obligación fuerte, pero esa obligación puede ser invalidada cuando el interés del paciente es más fuerte; la promesa de silencio per se no es excusa para la complicidad que el silencio encubre. Por otra parte, existe el problema moral de la justicia hacia el delatado, en cuyo juicio podemos equivocarnos; entonces vale preguntarse si el paciente tiene derecho a saber absolutamente todas las circunstancias que rodean el hecho que lo vulnera o si hay asuntos privados que no tiene derecho a saber, que acaso no sean estrictamente en su mejor interés, como el estado de salud o de discapacidad del cirujano.

\section{A MODO DE CIERRE}

Sopesar estos factores no es sencillo, el caso ideal es raro y el método cuestionable. Puede dudarse del interés público del acto denunciado, y aún más, se requiere que el reporte sea confidencial, no público, y que las conductas que de éste deriven sean exigibles y no meramente exhortativas. De tal suerte, delatar queda como último recurso. Además, es preciso considerar el daño colateral a otros actores, indirectamente involucrados en la iatrogenia, antes de fragilizar el sistema, antes de voltearlo contra uno para que después de la crisis, si la 
hay, se estabilice el statu quo. Por otra parte, la denuncia puede desatar en la percepción de los otros la posibilidad de una ganancia personal, dado el riesgo que existe de un mensaje sesgado, de modo que la sola posibilidad de ganancia mediante las revelaciones lleva a otros a cuestionar los motivos.

Queda siempre el rumor como recurso, aunque su eficacia es cuestionable. Satisface la pulsión de castigar pero causa cualitativamente los mismos daños que una denuncia y también puede revertirse contra el origen. En tanto rumor, quienes lo propagan no tienen ninguna obligación ni código de lealtad para no difundirlo, satisface otras pulsiones, aviva rencillas, rarifica el aire. Mina, pero no corrige.

Preguntar lo que uno haría depende de una escala de valores que no siempre coincide con valores ajenos. Resta sólo empeñarse en el buen comportamiento y las buenas maneras propias, sin ostentación. Cito al autor del texto hipocrático Sobre el médico (Peri letroy, ca. del año 350 o 300 a. de C.) "El hombre prudente debe también ser cuidadoso con ciertas consideraciones morales [...] debe tener el carácter de un caballero, así, debe ser serio y amable con todos, serio pero no severo, pues la severidad se toma como arrogancia [...] será honesto, porque la honestidad es de gran servicio [...] así entonces debiera ser el médico, en cuerpo y alma."11

Aplicar nuestros juicios y crítica duramente a nosotros mismos, pero acaso algo menos duramente a nuestros colegas, con la franqueza y la sinceridad de una filía, una simpatía; sin olvidar que del otro lado del triángulo hipocrático está el paciente, la otra filía.

\section{REFERENCIAS}

1. De Vega L. Fuente Ovejuna (ed. Rinaldo Froldi), 19a ed., Madrid, Espasa-Calpe (Colección Austral), 1998.

2. Acedo Castilla JF. El motín de Fuente Ovejuna en el teatro de Lope. La psicología de las muchedumbres y la naturaleza de sus delitos. En: Minervae Baeticae, Boletín de la Real Academia Sevillana de Buenas Letras. 2003; 31: 173-188.

3. Fumagalli G. Errare humanum est, perseverare autem diabolicum. En: Chi l'ha detto? 10a ed. Milán: Ulrico Hoepli; 1989. p. 326.

4. Bok S. Whistleblowing and Professional Responsibilities. En: Callahan D, Bok S. Ethics Teaching in Higher Education. Nueva York: Plenum Press; 1980. p. 277-295.

5. Sanford J. New policy to require evaluations for late-career practitioners. Stanford Medicine News. July 16, 2012. http://med.stanford.edu/news/allnews/2012/07/new-policy-to-require-evaluations-forlate-career-practitioners.html

6. Boodman SG. Aging doctors face greater scrutiny. Kaiser Health News. December 10, 2012. http://www. kaiserhealthnews.org/Stories/2012/December/11/ aging-doctors-face-greater-scrutiny.aspx

7. Waljee JF, Greenfield LJ. Aging and surgeon performance. Adv Surg. 2007; 41: 189-198.

8. Waljee JF, Greenfield LJ, Dimick JB, Birkmeyer JD. Surgeon age and operative mortality in the United States. Ann Surg. 2006; 244: 353-362.

9. Choudhry NK, Fletcher RH, Soumerai SB. Systematic review: the relationship between clinical experience and quality of health care. Ann Intern Med. 2005; 142: 260-273.

10. Plinio. Natural History. (tr. Rackham H.) Vol. II, Libro VII, Cap. 51, 168. Loeb Classical Library. Cambridge: Harvard University Press; 1961. p. 618-619.

11. The Physician. Cap. 1. En: Hippocrates. Vol. II. (tr. Jones WHS) Londres: William Heinemann Ltd.; 1923, reimp. 1959. p. 310-313.

Correspondencia: Alberto Campos

Unidad de Posgrado. Edificio A, primer piso. Circuito de Posgrados, Ciudad Universitaria, 04510, Ciudad de México. E-mail: alberto campos@hotmail.com https://orcid.org/0000-0001-5811-1908 